ErbB-2 structure

\title{
THE TRANSMEMBRANE DOMAIN OF THE ONCOGENIC \\ MUTANT ErbB-2 RECEPTOR: A STRUCTURE OBTAINED \\ FROM SITE-SPECIFIC INFRARED DICHROISM AND \\ MOLECULAR DYNAMICS
}

\author{
Andrew J. Beevers and Andreas Kukol* \\ Department of Biological Sciences, University of Warwick, Coventry, UK
}

Address correspondence to: Andreas Kukol, Department of Biological Sciences, University of Warwick, Gibbet Hill Road, Coventry, UK, CV4 7AL, Tel. +44 (0)24 7652 8373; Fax.+44 (0)24 7652 3701; Email: a.kukol@warwick.ac.uk

\section{SUMMARY}

ErbB-2 is a member of the family of epidermal growth factor receptors, which shows an oncogenic mutation in the rat gene neu, Val664Glu in the transmembrane domain that causes permanent dimerisation and subsequently leads to uncontrollable cell division and tumour formation. We have obtained the $\alpha$-helical structure of the mutant transmembrane domain dimer experimentally with site-specific infrared dichroism (SSID) based on six transmembrane peptides with ${ }^{13} \mathrm{C}^{18} \mathrm{O}$ carbonyl group labelled residues. The derived orientational data indicate a local helix tilt ranging from $28^{\circ} \pm 6^{\circ}$ to $22^{\circ} \pm 4^{\circ}$. Altogether using orientational constraints from SSID and experimental $\alpha$-helical constraints while performing a systematic conformational search including molecular dynamics simulation in a lipid bilayer, we have obtained a unique experimentally defined atomic structure. The resulting structure consists of a 
right handed $\alpha$-helical bundle with the residues Ile659, Val663, Leu667, Ile671, Val674 and Leu679 in the dimerisation interface. The right handed bundle is in contrast to the left-handed structures obtained in previous modelling efforts. In order to facilitate tight helical packing, the spacious Glu664 residues do not interact directly but with water molecules which enter the bilayer.

\section{KEYWORDS}

Neu, molecular modelling, $\alpha$-helical bundles, infrared spectroscopy, membrane proteins

\section{INTRODUCTION}

ErbB-2 is an epidermal growth factor receptor involved in the mediation of cell growth and differentiation ${ }^{1 ; 2}$. ErbB receptors are composed of an extracellular ligand binding region, a transmembrane region and a cytoplasmic tyrosine kinase. Activation of the tyrosine kinase occurs by ligand induced dimerisation, a generally accepted activation pathway for all receptor tyrosine kinases ${ }^{3}$. The ErbB-2 receptor in particular is activated by heteromeric association with other ErbB receptors which explains the fact that no high affinity ligand for ErbB-2 has been found ${ }^{4}$. The rat oncogene neu encodes a mutant ErbB-2 receptor, which contains a single Val664Glu mutation in the transmembrane domain ${ }^{5}$. This mutation causes permanent association and activation of ErbB-2 that leads finally to tumour formation ${ }^{6 ;} 7$. Previous experimental studies using NMR and infrared spectroscopy have provided evidence that the transmembrane domain of ErbB-2 is a dimeric $\alpha$-helical bundle and that the Glu664 residue is protonated and strongly hydrogen bonded ${ }^{8}$. Based on this observation a model has been presented that involves hydrogen bond mediated 
association of the two Glu residues as the major factor for permanent dimerisation of the mutant transmembrane domain ${ }^{8 ; 9}$.

However, several studies using molecular dynamics (MD) simulations have suggested that this interaction may lead to unfavourable packing of the two residues and that helix deformations are required to facilitate the hydrogen bonding thus disfavouring such a model ${ }^{10 ; 11}$. More recent studies using MD simulations have suggested that water molecules enter the bilayer and interact with Glu residues and that the transmembrane domain shifts position to allow interaction of these residues with water $^{12 ; 13 ; 14}$.

Ultimately, only high resolution structural studies using the transmembrane domain in lipid bilayers are able to resolve these discrepancies. However, applications of highresolution techniques such as x-ray crystallography and NMR spectroscopy to membrane proteins face enormous difficulties resulting in far fewer known membrane protein structures compared to water-soluble proteins. Here we use the novel method of site-specific infrared dichroism (SSID) ${ }^{15 ; 16}$ combined with a systematic conformational search using experimentally constrained MD simulations for the first time in a lipid bilayer/water system. This method has been successfully employed in previous studies using in vacuo MD simulations for example for the M2 proton channel of the Influenza A virus ${ }^{17}$, the $\mathrm{CM} 2$ domain of the Influenza $\mathrm{C}$ virus ${ }^{18}$ and the mammalian MHC associated invariant chain ${ }^{19}$. The SSID method involves the use of several synthetic peptides, in which one residue has a ${ }^{13} \mathrm{C}={ }^{18} \mathrm{O}$ labelled carbonyl bond ${ }^{20}$. The peptides are incorporated into liposomes, which are studied as oriented lipid multilayers with attenuated total reflection Fourier-transform infrared spectroscopy (ATR-FTIR). The helix tilt and rotational pitch angle of a particular ${ }^{15 ; 21}$ 
residue obtained from SSID are used as additional energy terms in MD simulations systematically exploring the helix-helix interaction.

\section{RESULTS}

FTIR measurements - Typical FTIR spectra of the amide I region for each labelled peptide are shown in figure 2. Please note that no band sharpening technique like Fourier self-deconvolution has been applied. As can be clearly seen, the amide I absorption band at $1658 \mathrm{~cm}^{-1}$ is a symmetrical and non-shouldered peak, indicating that a predominantly $\alpha$-helical structure is adopted by the peptide in the lipid bilayer environment. Residual intensity at $1618 \mathrm{~cm}^{-1}$ corresponds to ${ }^{13} \mathrm{C}={ }^{16} \mathrm{O}$ labelled carbonyl bonds, because the amino acid does not exchange to $100 \%$ with $\mathrm{H}_{2}{ }^{18} \mathrm{O}$. The absorption band at $1594 \mathrm{~cm}^{-1}$ represents the ${ }^{13} \mathrm{C}={ }^{18} \mathrm{O}$ label ${ }^{20 ; 22}$, confirming that the labelling procedure was successful in all cases. As shown in figure 2, spectra were recorded at infrared radiation polarised parallel and perpendicular with respect to the plane of incidence, which produced a dichroic ratio in the range of $\mathrm{R}=2.4$ to 4.2 for the amide I absorption band, depending on sample order, and in the range of $\mathrm{R}=2.4$ to 19.6 for the isotopic label absorption band, depending on position and sample order. In each instance, the dichroic ratio for the isotopic label was different from the amide I peak indicating oligomerisation. Table 1 shows the resultant orientational data as defined in Figure 1A calculated from the SSID theory ${ }^{15 ; 21}$. There is a tendency of variation in the local tilt $\beta$ of the helix along the peptide strand, being $\beta=28^{\circ} \pm 6$ at Ile659/Ile660 and $\beta=22^{\circ} \pm 4^{\circ}$ at the Val675/676 labels, although the variation is within the experimental error. At positions closer to the centre of the peptide strand (Leu667/Leu668) the helix adopts a local tilt of $\beta=25^{\circ} \pm 8^{\circ}$. 
Molecular Dynamics Conformational Searching - MD simulations of 36 different rotational orientations with left-handed and right-handed crossing angle and simulation at four different random initial atom velocities produced 288 different peptide structures in a DMPC lipid bilayer/water system. Figure 3 shows the resulting rotations of the initial structures and their respective energies following MD simulation. Clustering into categories of similar structures (as described in experimental procedures) followed by averaging produced seven structures, of which two were energetically unstable (figure 3). Of the remaining five structures, structure 5 has the closest correlation to the experimental rotational pitch angles from the infrared data (table 2). Altogether eight substructures contributed to this cluster average, which is shown in figure 4 . The $\alpha$-helices remain close together although no interhelical distance restraints have been applied during the simulation. The structure in figure 4 reveals a right-handed $\alpha$-helical bundle with a crossing angle of $\sim 50^{\circ}$. The structure is tightly packed, displaying the classic 'knobs into holes' arrangement (figure 4B) ${ }^{23}$. The dimerisation interface between the helices is composed of Ile659, Val663, Leu667, Ile671, Val674 and Leu679. The bulky aromatic Phe669 groups are on the exterior of the complex, minimising steric hindrance and allowing tight packing to occur. By comparison, the Phe658 groups are near to the interhelical interface, but their arrangement does not appear to cause deformation of the helices. There also appears to be an interaction between one Thr662 residue and the backbone carbonyl oxygen of the Phe658 residue of the same helix. There is no direct interaction between Glu residues, as they are not in such an arrangement, but instead an interaction between these side-chains and water molecules, which have entered the bilayer during simulation, was observed (figure 4C). No other water molecules were capable of entering the lipid region other than those interacting with Glu residues. 


\section{ErbB-2 structure}

\section{DISCUSSION}

The FTIR data clearly indicates that the ErbB-2 peptide is in an $\alpha$-helical conformation and the labelled sites also adopt this particular secondary structure (indicated by their absorbance at $1594 \mathrm{~cm}^{-1}$ ). Dichroic ratios vary in each FTIR spectrum for both the amide I peak and for the label peak. For the amide I peak these differences are solely the result of random variations in the sample order (which is taken into account in the calculation of the orientations ${ }^{21}$ ). For the label, the extra factor of position of the ${ }^{13} \mathrm{C}={ }^{18} \mathrm{O}$ bond must be taken into account (as different positions will have different tilts and rotations). The difference in the dichroic ratios of the amide I peak and the labelled peak indicate oligomerisation. The variations of the helix tilt are almost within the margin of error, although a trend for lower helix tilts from $\mathrm{N}$ to $\mathrm{C}$ terminus may be established.

Following the constrained MD search, the resulting structure does not show the presence of inter-helical Glu hydrogen bonds. The interaction seen in figure 4 involves water molecules, which interact with the Glu residues. The rotation at which this interaction can occur also appears to allow tight helical packing, as the structure corresponds fairly well to the conventional 'knobs-into-holes' pattern of $\alpha$-helical packing ${ }^{23}$. This is in accordance with previous unconstrained molecular simulation studies, which suggest that the direct Glu-Glu interaction is incompatible with favourable packing ${ }^{10 ; 11}$. NMR studies using glutamine residues in place of the Glu have also indicated that the direct interaction is probably not adopted ${ }^{24}$.

The adopted rotation may be a balance between these two factors; as the Phe658 residues are allowed to stack without causing disruption to the helical geometry, whilst Phe669 residues can point away from the complex. The interfacial motif observed is fairly typical of helical packing in a lipid bilayer and will allow tight 


\section{ErbB-2 structure}

packing to occur. At the same time, both the Glu664 and Thr662 residues are able to form hydrogen bonds, thereby stabilising the polar groups in their apolar environment. The other previously suggested rotation, that of interaction between Glu residues and the carbonyl backbone of the opposing peptide chain ${ }^{9}$, is not observed either. This requires a different rotational pitch angle to that adopted by the structure obtained in this study. Neither is there any evidence in the structure of significant deformation of the $\alpha$-helical complex, suggested by previous study as a means to allow potential sites for Glu hydrogen bonding interactions ${ }^{10}$.

Water molecules have been previously suggested as an important factor in the dimerisation of mutant ErbB-2 by long MD simulations on selected rotations of the complex $12 ; 13 ; 14$. It is still unclear exactly how or why water molecules enter the bilayer. But previous research has suggested that the $\alpha$-helical complex itself also shifts position slightly in order to move the Glu residues closer to the water molecules and the polar regions of the lipid molecules. This should increase the likelihood of such an interaction occurring.

It is still unclear exactly how the Glu residue mutation results in permanent dimerisation of the complex. One possibility is the presence of a different conformation in a fluid-state hydrated bilayer, as opposed to the partially dry gel-state bilayer studied in the experiment (the bilayer in the MD simulation is in the fluidstate).

Another possibility is that wild-type and mutated ErbB-2 have different dimerisation motifs and the Glu has some role to play in stabilising the mutated dimerisation, such as the water interaction. This is supported by recent experimental studies which indicate that the Glu mutation has little or no effect on the dimerising ability of ErbB- ${ }^{25}$. A proposed reason for this is that the Glu mutation allows the helix to 


\section{ErbB-2 structure}

adopt different orientations to those adopted by the wild-type, and it is this that promotes constant dimerisation. There also has been the suggestion that the wild-type ErbB-2 possesses two dimerisation motifs, an active and inactive form, and that the mutation shifts the equilibrium towards the active form ${ }^{26}$.

In order to explore these hypotheses further, the transmembrane domain of the wildtype ErbB-2 peptide would need to be structurally elucidated in a similar way. This is practically more difficult, if extracellular ligand binding is required for dimerisation, although there is some evidence that the wild-type transmembrane domain can selfassociate without its extracellular region ${ }^{25}$.

The right-handed crossing angle between the $\alpha$-helices is in contrast to previous MD simulation studies, which have suggested a left-handed crossing for the mutant ErbB-2 complex ${ }^{10 ; 11}$. However, these studies did not include experimental restraints. Some left-handed structures were produced in our own study, but none of these fit the infrared data as well as structure 5. Right-handed crossing was observed using two interhelical distance constraints between ${ }^{13} \mathrm{CH}_{2}$ Gly665 on helix 1 and ${ }^{13} \mathrm{C}=\mathrm{O}$ Glu664 and ${ }^{13} \mathrm{CH}_{2}$ Gly665 on helix 2 from solid-state NMR studies with an in vacuo minimisation and molecular dynamics procedure ${ }^{24}$, although the dimerisation motif is different in this study. We analysed the model produced from this study and obtained rotational pitch angles of $\omega=74^{\circ}$ for Ile 659 and $\omega=155^{\circ}$ for Leu667 sites, which differ from our own IR data by $150^{\circ}$ and $163^{\circ}$ respectively (the solid-state NMR model does not include the segment containing the Val675 residue). Key differences between this study and our own work include the mutation of Glu to Gln, which can alter the preferred orientation due to different side-chain interactions. Additionally the simulation in the previous study was performed without explicit representation of lipid and water molecules, which may also affect the results. 


\section{ErbB-2 structure}

The role of the lipid membrane itself also requires further investigation. The DMPC lipid bilayer used in this study has been used in all previously reported MD simulation studies $11 ; 12 ; 13 ; 14$ (apart from those performed in vacuo ${ }^{10}$ ). DMPC was also the bilayer of choice in earlier NMR studies ${ }^{8}$, although more recent work has looked at the protein in a lipid bilayer composed of the partially unsaturated POPC molecules ${ }^{24}$. The composition of the bilayer may have an effect on the stability and orientation of the transmembrane peptide complex, both sterically and energetically. Naturally occurring membranes possess various types of lipid chains and ideally should be used for such studies, but so far only MD studies focussing on binary mixtures begin to emerge.

\section{CONCUSIONS}

This is the first study of the mutant ErbB-2 transmembrane domain to yield a high resolution structure based on experimental constrains and MD simulations in a lipid bilayer/water system. This research demonstrates the necessity to combine simulation and experiment in order to investigate the structure of transmembrane helical bundles. This allows for the inclusion of specific experimental data, directly relevant to the system in question, into the computational simulations.

Furthermore, this work demonstrates the usefulness of a conformational search in a lipid bilayer which was here attempted for the first time compared to earlier in vacuo searches ${ }^{10}$. But it is clear that, even with explicit representation of solvent and lipid molecules, energetics alone cannot accurately determine the actual structure of the domain in question. Only by combining simulation and experimental data such as site-specific IR dichroism can one hope to produce accurate models out of many energy minima resulting from experimentally unconstrained simulation. 
This work should support further modelling research of the full length ErbB-2 receptor assembling the known crystal structures of the extracellular and intracellular domains ${ }^{27}$ with the obtained transmembrane domain structure in order to understand the biology of Erb-B receptors in disease processes.

\section{MATERIALS AND METHODS}

Amino Acid Labelling $-1-{ }^{13} \mathrm{C}$ amino acids were obtained from Cambridge Isotope Laboratories (Cambridge, MA) and labelled with ${ }^{18} \mathrm{O}$ at the carboxyl group using $\mathrm{H}_{2}{ }^{18} \mathrm{O}$ (95\% enrichment - Campro Scientific, Veenendaal, Holland) at low $\mathrm{pH}$ as described elsewhere ${ }^{20}$. The 9-fluorenylmethyl-oxycarbonyl (Fmoc) protecting group has been added following literature procedures ${ }^{28}$ by reaction with Fmoc-ONSu using triethylamine as a base (sodium hydroxide in the case of leucine). The products of each reaction step were analysed by mass spectrometry.

Peptide Synthesis - Six peptides of the transmembrane domain of ErbB-2 (residues 652-685 RASPVTFIIATVEGVLLFLIL VVVVGILIKRRR) were obtained from Keck Laboratories (Yale University, USA) with one ${ }^{13} \mathrm{C}={ }^{18} \mathrm{O}$ labelled amino acid residue each in the positions indicated in boldface italic in the sequence. The resulting peptides were purified as described elsewhere for analogous transmembrane peptides 19; 29 . Briefly, the peptide was dissolved in trifluoroacetic acid and purified by reversed phase chromatography (Jupiter 5C4-300 Å column, Phenomenex, Cheshire, UK). Peptide elution was achieved with a linear gradient to a final solvent composition of $5 \% \mathrm{H}_{2} \mathrm{O}, 38 \%$ acetonitrile and 57\% 2-propanole. All solvents contained $0.1 \%(\mathrm{v} / \mathrm{v})$ trifluoroacetic acid. After lyophilisation of the pooled fractions 
ErbB-2 structure

the peptide was dissolved in 1,1,1,3,3,3-hexafluoro-2-propanol, containing dimyristoylphosphocholine (Sigma, UK), to a peptide to lipid ratio of 1:30 (w/w)

Preparation of Proteoliposomes - Proteoliposomes were prepared by rotational evaporation of the organic solvent followed by addition of water to obtain a lipid concentration of $10 \mathrm{mg} / \mathrm{mL}$. Liposome preparations were then sonicated and freezethawed three times and stored frozen. The $\mathrm{pH}$ of the resulting preparations was approximately 4 .

Attenuated Total Reflection Fourier Transform Infrared Spectroscopy Approximately $120 \mu \mathrm{L}$ of proteoliposome suspension was deposited onto a trapezoidal Germanium internal reflection element (50 mm x $2 \mathrm{~mm}$ x $20 \mathrm{~mm}$ ). After the removal of bulk water in a stream of nitrogen the FTIR spectrum was taken as an average of 1000 interferograms using a Bruker Tensor FTIR spectrometer (Bruker Optics, Coventry, UK) containing a high sensitivity liquid nitrogen-cooled MCT/A detector. Each spectrum was taken in the 4000 to $1000 \mathrm{~cm}^{-1}$ range of the electromagnetic radiation at a resolution of $2 \mathrm{~cm}^{-1}$ per data point. Spectra were recorded at parallel and perpendicular polarisation and the dichroic ratio was calculated as the ratio between the integrated absorption of parallel and perpendicular polarised light for the amide I absorption band centred at wavenumber $1658 \mathrm{~cm}^{-1}$ and for the ${ }^{13} \mathrm{C}={ }^{18} \mathrm{O}$ absorption band centred at $1594 \mathrm{~cm}^{-1}$. For each of the six peptides at least three independent measurements were taken. Altogether twenty-five spectra were obtained and analysed. 


\section{ErbB-2 structure}

Data Analysis - The resulting dichroic ratios were analysed for each pair of adjacent labels using the theory of (SSID) as presented initially by Arkin et al. (1997) ${ }^{15}$ with modifications by Kass et al. (2004) ${ }^{16}$ resulting in the local helix tilt $\beta$ and the rotational pitch angle $\omega$ of the labelled carbonyl bond (for definition of the geometrical parameters see figure 1). Briefly, the theory of SSID allows to deduce the orientational parameters of a peptide $\alpha$-helix from the experimental dichroic ratio of the unlabelled amide I absorption band $\left(\mathrm{R}_{\text {helix }}\right)$ and the dichroic ratio of the absorption band caused by the labelled residue $\left(\mathrm{R}_{\text {site }}\right) . \mathrm{R}_{\text {helix }}$ is a function of the helix tilt angle $\beta$ and the distribution around this tilt angle caused by sample disorder and mosaicity. $\mathrm{R}_{\text {site }}$ depends additionally on the rotational pitch angle $\omega$. In order to obtain the geometrical parameters $\beta$ and $\omega$ an additional label is required at a position next in sequence such that the rotational pitch angle is $\omega+100^{\circ}$ assuming a canonical $\alpha$-helix, which is a valid assumption over such a short distance. The four resulting non-linear equations can be solved by standard numerical methods.

Note that contrary to the conventional analysis of dichroic ratios ${ }^{30}$ an exact helix tilt can be obtained, even if the sample is partially disordered, while conventional analysis only yields a maximum tilt angle. The angle $\theta$ between the labelled $\mathrm{C}=\mathrm{O}$ bond and the $\mathrm{z}$-axis was calculated from the equation ${ }^{21}$ :

$$
\cos \theta=\cos \alpha \cos \beta-\sin \alpha \sin \beta \cos \left(\omega+17^{\circ}\right)
$$

Where $\alpha$ is defined as the angle between the transition dipole moment and the molecular director, given as $38^{\circ}$ in $\alpha$-helices ${ }^{31}$. All calculations were carried out with Mathematica 5 (Wolfram Research, Champaign, IL). Typically seven results were averaged. 
Systematic Molecular Dynamics (MD) Searching - The conformational search and simulations were performed using the CHI suite of programs ${ }^{32}$ for CNS-SOLVE ${ }^{33}$ combined with the GROMACS molecular simulation software ${ }^{34 ; 35}$. CHI was used to create dimeric starting structures by rotating the helices through $10^{\circ}$ increments for both left and right-handed dimers (crossing angle $\pm 25^{\circ}$ ). Each structure was created four times (to allow MD simulations with 4 different random velocities) to produce a total of 288 starting structures. GROMACS was then used to perform MD simulations at $300 \mathrm{~K}$. Each starting structure was placed in a pre-equilibrated bilayer of 128 DMPC molecules ${ }^{36}$. In-house developed software was then used to remove lipid molecules overlapping with the protein within a distance of $0.03 \AA$. A hole within the bilayer was then created using 30ps of MD simulation using an outwards directed force on the lipid molecules ${ }^{37}$. The bilayer was orientated such that the lipid/water interface was perpendicular to the defined z-axis of the overall system. Ten chloride counter-ions were added to neutralize the overall charge of the system and the resulting bilayer was then subjected to 100 ps of MD with the peptide atoms restrained in their current positions, followed by unrestrained energy minimisation. The system was then subjected to 200 ps of unrestrained molecular dynamics followed by energy minimisation. Simulations were performed with the GROMACS87 forcefield ${ }^{38}$ containing additional parameters for lipid molecules ${ }^{39 ; 40}$. Bond lengths were constrained with the LINCS algorithm ${ }^{41}$ allowing a time-step of $2 \mathrm{fs}$. Temperature and pressure were controlled using the Berendsen coupling scheme with a time constant of $0.1 \mathrm{ps}$ for both temperature coupling and pressure coupling ${ }^{42}$. All simulations were carried out with periodic boundary conditions. Electrostatic interactions were treated with a twin cut-off range of $1 \mathrm{~nm} / 1.8 \mathrm{~nm}$ and van der Waal's interactions were cut-off at $1 \mathrm{~nm}$. 
Each simulation was carried out with experimental constraints on the angle of the labelled $\mathrm{C}=\mathrm{O}$ carbonyl bonds to the z-axis with a force constant of $5000 \mathrm{~kJ} \mathrm{~mol}^{-1}$. Local tilts were also taken into account by constraining the angle to the z-axis of a vector defined by the $\mathrm{C} \alpha$ atoms of the labelled residue at position $\mathrm{i}$ and residue at the relative $i+7$ position. Further experimental constraints accounted for the $\alpha$-helical secondary structure of the peptide (confirmed by our FTIR and previous experiments ${ }^{8}$ ) by allowing distances between carbonyl oxygens and amide hydrogens of residues $\mathrm{i}$ and $\mathrm{i}+4$ in the transmembrane region to vary freely between $1.6 \mathrm{~nm}$ and $2.3 \mathrm{~nm}$ and applying a harmonic potential outside these boundaries. Altogether twenty-nine constraints were placed on each of the peptides.

Once the search had been performed, $\mathrm{C} \alpha$ root-mean-square-deviation (RMSD) comparisons were made between all resulting structures. Structures were then clustered according to their RMSD values. The adopted criteria for structure clustering were that of a maximum RMSD value of $2 \AA$ between two structures. For a cluster to be created, a minimum of five structures possessing RMSD values within this limit was required.

The structures contributing to this cluster were then used to create an average structure, which was subjected to MD simulation for $2 \mathrm{~ns}$ in the DMPC, followed by energy minimisation.

\section{ACKNOWLEDGEMENTS}

We would like to express our thanks to Sue Slade and Charlotte Winn of the University of Warwick Proteomics group for mass spectrometry analysis, to Steven O. Smith for kindly providing the atomic coordinates of his model of the neu/ErbB-2 transmembrane domain and to Teresa Pinheiro for generous access to the FTIR 
ErbB-2 structure

spectrometer funded by the Medical Research Council UK (grant no.G9901445).This work was funded by the Biotechnology and Biological Sciences Research Council UK (grant no. 88/B19450). 


\section{REFERENCES}

1. Leahy, D. J. (2004). Structure and Function Of The Epidermal Growth Factor (EGF/ErbB) Family Of Receptors. Advances in Protein Chemistry 68, 1-27.

2. Olayioye, M. A., Neve, R. M., Lane, H. A. \& Hynes, N. E. (2000). The ErbB signalling network: receptor heterodimerization in development and cancer. EMBO Journal 19, 3159-3167.

3. Schlessinger, J. (2000). Cell Signalling by Receptor Tyrosine Kinases. Cell 103, 211-225.

4. Klapper, L. H., Glathe, S., Vaisman, N., Hynes, N. E., Andrews, G. C., Sela, M. \& Yarden, Y. (1999). The ErbB-2/HER2 oncoprotein of human carcinomas may function solely as a shared coreceptor for multiple stromaderived growth factors. Proceedings of the National Academy of Sciences USA 96, 4995-5000.

5. Bargmann, C. I., Hung, M.-C. \& Weinberg, R. A. (1986). Multiple independent activations of the neu oncogene by a point mutation altering the transmembrane domain of p185. Cell 45, 649-657.

6. Bargmann, C. I. \& Weinberg, R. A. (1988). Increased Tyrosine Kinase Activity Associated with the Protein Encoded by the Activated neu Oncogene. Proceedings of the National Academy of Sciences USA 85, 5394-5398.

7. Bargmann, C. I. \& Weinberg, R. A. (1988). Oncogenic activation of the neuencoded receptor protein by point mutation and deletion. EMBO Journal 7, 2043-2052.

8. Smith, S. O., Smith, C. S. \& Bormann, B. J. (1996). Strong hydrogen bonding interactions involving a buried glutamic acid in the transmembrane sequence of the neu/erbB-2 receptor. Nature Structural Biology 3, 252-258.

9. Sternberg, M. J. E. \& Gullick, W. J. (1989). Neu Receptor Dimerization. Nature 339, 587.

10. Sajot, N. \& Genest, M. (2000). Structure prediction of the dimeric neu/ErbB-2 transmembrane domain from multi-nanosecond molecular dynamics simulations. European Biophysics Journal 28, 648-662.

11. Aller, P., Voiry, L., Garnier, N. \& Genest, M. (2005). Molecular Dynamics (MD) Investigations of Preformed Structures of the Transmembrane Domain of the Oncogenic Neu Receptor in a DMPC Bilayer. Biopolymers 77, 184-197.

12. Soumana, O. S., Aller, P., Garnier, N. \& Genest, M. (2005). Transmembrane Peptides from Tyrosine Kinase Recptor. Mutation-related behaviour in a Lipid Bilayer Investigated by Molecular Dynamics Simulations. Journal of Biomolecular Structure and Dynamics 23, 91-99.

13. Garnier, N., Crouzy, S. \& Genest, M. (2003). Molecular Dynamics Simulations of the Transmembrane Domain of the Oncogenic ERbB2 Receptor Dimer in a DMPC Bilayer. Journal of Biomolecular Structure and Dynamics 21, 179-199.

14. van der Ende, B. M., Sharom, F. J. \& Davis, J. H. (2004). The transmembrane domain of Neu in a lipid bilayer: molecular dynamics simulations. European Biophysics Journal 33, 596-610. 
15. Arkin, I. T., MacKenzie, K. R. \& Brünger, A. T. (1997). Site-directed dichroism as a method for obtaining rotational and orientational constraints for orientated polymers. Journal of the American Chemical Society 119, 89738980.

16. Kass, I., Arbely, E. \& Arkin, I. T. (2004). Modeling sample disorder in sitespecific dichroism studies of uniaxial systems. Biophysical Journal 86, 25022507.

17. Kukol, A., Adams, P. D., Rice, L. M., Brunger, A. T. \& Arkin, I. T. (1999). Experimentally Based Orientational Refinement of Membrane Protein Models: A Structure for the Influenza A M2 H+ Channel. Journal of Molecular Biology 286, 951-962.

18. Kukol, A., Torres, J. \& Arkin, I. T. (2000). Use of a single glycine residue to determine tilt and orientation of a transmembrane helix. A new structural label for infrared spectroscopy. Biophysical Journal 79, 3139-3143.

19. Kukol, A., Torres, J. \& Arkin, I. T. (2002). A Structure for the trimeric MHC Class II-associated Invariant Chain Transmembrane Domain. Journal of Molecular Biology 320, 1109-1117.

20. Torres, J., Kukol, A., Goodman, J. M. \& Arkin, I. T. (2001). Site-specific examination of secondary structure and orientation determination in membrane proteins: the peptidic ${ }^{13} \mathrm{C}={ }^{18} \mathrm{O}$ group as a novel infrared probe. Biopolymers 59, 396-401.

21. Kukol, A. (2005). Site-specific IR spectroscopy and molecular modelling combined towards solving transmembrane protein structure. Spectroscopy 19, $1-16$.

22. Torres, J., Adams, P. D. \& Arkin, I. T. (2000). Use of a new label, 13C18O, in the determination of a structural model of phospholamban in a lipid bilayer. Spatial restraints resolve the ambiguity arising from interpretations of mutagenesis data. Journal of Molecular Biology 300, 677-685.

23. Crick, F. H. C. (1953). The Packing of Alpha-Helices: Simple Coiled-Coils. Acta Crystallographa 6, 689.

24. Smith, S. O., Smith, C. S., Shekar, S., Peersen, O., Ziliox, M. \& Aimoto, S. (2002). Transmembrane Interactions in the Activation of the Neu Receptor Tyrosine Kinase. Biochemistry 41, 9321-9332.

25. Mendrola, J. M., Berger, M. B., King, M. C. \& Lemmon, M. A. (2002). The Single Transmembrane Domains of ErbB Receptors Self-Associate in Cell Membranes. Journal of Biological Chemistry 277, 4704-4712.

26. Feishman, S. J. \& Ben-Tal, N. (2002). A putative molecular-activation switch in the transmembrane domain of erbB2. Proceedings of the National Academy of Sciences USA 99, 15937-15940.

27. Bagossi, P., Horvath, G., Vereb, G., Szollosi, J. \& Tozser, J. (2005). Molecular Modelling of Nearly Full-Length ErbB2 Receptor. Biophysical Journal 88, 1354-1363.

28. Kortenaar, P. B. W., Van Dijk, B. G., Peeters, J. M., Raaben, B. J., Adams, P. J. H. M. \& Tesser, G. I. (1986). Rapid and efficient method for the preparation of Fmoc-amino acids starting from 9-fluorenylmethanol. International Journal of Peptide and Protein Research 27, 398-400.

29. Kukol, A. \& Arkin, I. T. (1999). vpu Transmembrane Peptide Structure Obtained by Site-Specific Fourier Transform Infrared Dichroism and Global Molecular Dynamics Searching. Biophysical Journal 77, 1594-1601. 
30. Goormaghtigh, E., Raussens, V. \& Ruysschaert, J.-M. (1999). Attenuated total reflection infrared spectroscopy of proteins and lipids in biological membranes. Biochimica et Biophysica Acta 1422, 105-185.

31. Tsuboi, M. (1962). Infrared dichroism and molecular conformation of $\alpha$-form poly-gamma-benzyl-L-glutamate. Journal of Polymer Science 59, 139-153.

32. Adams, P. D., Arkin, I. T., Engelman, D. M. \& Brünger, A. T. (1995). Computational searching and mutagenesis suggest a structure for the pentameric transmembrane domain of phospholamban. Structural Biology 2, 154-162.

33. Brunger, A. T., Adams, P. D., Clore, M., DeLano, W. L., Gros, P., GrosseKunsteleve, R. W., Jiang, J. S., Kuszewski, J., Nilges, M., Pannu, N. S., Read, R. J., Rice, L. M., Simonson, T. \& Warren, G. L. (1998). Crystallography \& NMR System: A New Software Suite for Macromolecular Structure Determination. Acta Crystallographa D54, 905-921.

34. Berendsen, H. J. C., van der Spoel, D. \& van Drunen, R. (1995). GROMACS: A message passing parallel molecular dynamics implementation. Comp. Phys. Comm. 91, 43-56.

35. Lindahl, E., Hess, B. \& van der Spoel, D. (2001). Gromacs 3.0: A package for molecular dynamics simulation and trajectory analysis. J. Mol. Mod. 7, 306317.

36. Gurtovenko, A. A., Patra, M., Karrttunen, M. \& Vattulainen, I. (2004). Cationic DMPC/DMPAT Lipid Bilayers: Molecular Dynamics Study. Biophysical Journal 86, 3461-3472.

37. Faraldo-Gomez, J. D., Smith, G. R. \& Sansom, M. S. P. (2002). Setting up and optimisation of membrane protein simulations. European Biophysical Journal 31, 217-227.

38. Van Gunsteren, W. F. \& Berendsen, H. J. C. (1987). Gromos-87 Manual.

39. Tieleman, D. P. \& Berendsen, H. J. C. (1996). Molecular dynamics simulations of a fully hydrated dipalmitoylphosphatidylcholine bilayer with different macroscopic boundary conditions and parameters. Journal of Chemical Physics 105, 4871-4880.

40. Berger, O., Edholm, O. \& Jahnig, F. (1997). Molecular Dynamics simulations of a fluid bilayer of dipalmitoylphosphatidylcholine at full hydration, constant pressure and constant temperature. Biophysical Journal 72, 2002-2013.

41. Hess, B., Bekker, H., Berendsen, H. J. C. \& Fraajie, G. E. M. (1997). LINCS: A Linear Constraint Solver for Molecular Simulations. Journal of Computational Chemistry 18, 1463-1472.

42. Berendsen, H. J. C., Postma, J. P. M., Van Gunsteren, W. F., DiNola, A. \& Haak, J. R. (1984). Molecular Dynamics with coupling to an external bath. Journal of Chemical Physics 81, 3684-3690.

43. Humphrey, W., Dalke, A. \& Schulten, K. (1996). VMD: Visual Molecular Dynamics. Journal of Molecular Graphics 14, 33-38. 
ErbB-2 structure

\section{FOOTNOTES}

\# Abbreviations Used: FMOC - 9-flourenylmethoxylcarbonyl; DMPC dimyristoylphosphocholine; FTIR - Fourier transform infrared; NMR - Nuclear Magnetic Resonance; RMSD - Root Mean Square Deviation, SSID - site specific infrared dichroism. 
ErbB-2 structure

\section{FIGURES}

Figure 1: (A) Definition of the helix tilt $\beta$, the rotational pitch angle $\omega$ and the angle $\theta$ representing the angle between a particular labelled amide I transition dipole moment and the z-axis. $\omega$ is defined as $0^{\circ}$ when the transition dipole moment points in the direction of the helix tilt. The angle $\alpha$ is defined as $38^{\circ}$ for all $\alpha$-helices ${ }^{31}$. (B) The method of rotating the peptides by increments of the angle $\phi$, resulting in the array of starting structures used for MD simulation. The angle $\Omega$ represents the helical crossing angle.

Figure 2: Infrared spectra for each peptide containing a ${ }^{13} \mathrm{C}={ }^{18} \mathrm{O}$ label in the position indicated in the top left. The amide I area is displayed, while the insert shows the magnified amide I absorption band of the label.

Figure 3: (A) Polar plot of the energies of structures obtained from MD simulation of mutant ErbB-2 in dependence of the helix rotation angle $\phi$. The distance from the centre indicates negative energy $(\mathrm{E})$ in $\mathrm{kJ} / \mathrm{mol}$. Each individual structure is indicated by a triangle, while the clustered averages are shown as numbered circles. The arcs represent the movement of structures from their starting positions with respect to the helix rotation angle $\phi$ during the MD simulation. (B) The rotation of the helix dimer defined as $\phi=0$.

Figure 4: Structure of the ErbB-2 transmembrane domain obtained by SSID constrained MD simulations. (A) The structure in ribbon representation with Glu residues displayed. (B) Spacefill rendering of structure, Glu in black and labelled residues in dark grey. (C) A detailed view of the Glu interaction with water molecules. 


\section{ErbB-2 structure}

(D) View of the Glu residue packing from top of helix. All figures have been created with $\mathrm{VMD}^{43}$. 


\section{ErbB-2 structure}

\section{TABLES}

Table 1: Local helix tilt angle $\beta$, rotational pitch angle $\omega$ and the resulting calculated angle of $\mathrm{C}=\mathrm{O}$ bond to $\mathrm{z}$-axis $\theta$, from dichroic ratio comparisons between peptides with neighbouring labels.

$\begin{array}{llll}\text { Label } & \beta & \theta & \omega\end{array}$

$\begin{array}{cccc}\text { Ile659/Ile660 } & 28.1^{\circ} \pm 6^{\circ} & 56^{\circ} \pm 3^{\circ} & 284^{\circ} \pm 8^{\circ} \\ \text { Leu667/Leu668 } & 25.2^{\circ} \pm 8^{\circ} & 63^{\circ} \pm 6^{\circ} & 318^{\circ} \pm 14^{\circ} \\ \text { Val675/Val676 } & 21.9^{\circ} \pm 4^{\circ} & 59^{\circ} \pm 2^{\circ} & 327^{\circ} \pm 10^{\circ}\end{array}$




\section{ErbB-2 structure}

Table 2: Comparison of rotational pitch angles obtained from infrared spectra to those possessed by the clustered average structures. The deviation of the rotation angles from the experimental values for each averaged structure is also shown for comparative purposes. This is calculated using the formula $\sqrt{\sum\left(\omega_{\exp }-\omega_{\bmod e l}\right)^{2}}$.

\begin{tabular}{ccccccc} 
Label & $\omega_{\text {exp }}$ & 1 & 2 & 3 & 4 & 5 \\
\hline Ile659 & $284^{\circ}$ & $275^{\circ}$ & $152^{\circ}$ & $267^{\circ}$ & $255^{\circ}$ & $\mathbf{2 5 5 ^ { \circ }}$ \\
Leu667 & $318^{\circ}$ & $93^{\circ}$ & $313^{\circ}$ & $326^{\circ}$ & $344^{\circ}$ & $\mathbf{3 2 9}^{\circ}$ \\
Val675 & $327^{\circ}$ & $203^{\circ}$ & $16^{\circ}$ & $69^{\circ}$ & $72^{\circ}$ & $\mathbf{3 0 7 ^ { \circ }}$ \\
Deviation & & $184^{\circ}$ & $142^{\circ}$ & $104^{\circ}$ & $112^{\circ}$ & $\mathbf{3 8}^{\circ}$
\end{tabular}

